\title{
TDAH Y SU RELACIÓN CON LA MOTIVACIÓN EN EL CONTEXTO EDUACTIVO
}

Gómez Díaz, Isabel C.; Morán Alvarado, Verónica; Pereda Moral, Macarena; Pazos

Moreno, Eloísa. Universidad de Huelva

\section{Resumen}

Puesto que el objetivo de este trabajo es conocer la influencia de la motivación en los sujetos con Trastorno por Déficit de Atención con Hiperactividad (TDAH), se ha revisado la conceptualización del trastorno en la historia y su relación con la motivación, para determinar si tras el déficit atencional subyacen problemas motivacionales. El artículo señala las variables o factores más relevantes en el desarrollo del trastorno, éstas pueden ser genéticas, psicobiológicas y ambientales, desde una perspectiva más general. Tras analizar tanto la etiología del término como las variables que determinan el trastorno y la posible relación motivacional, se proponen una serie de estrategias educativas para fomentar el rendimiento académico de niños con TDAH. En definitiva, este trabajo manifiesta una posible relación directa entre atención y motivación, lo que marcará el desarrollo curricular de los alumnos en el contexto educativo.

Palabras claves: atención, aprendizaje activo, motivación, estrategias educativas.

\section{Introducción}

El Trastorno por Déficit de Atención e Hiperactividad (TDAH) se caracteriza por un patrón persistente de inatención, exceso de actividad e impulsividad como características definitorias. Según el Manual Diagnóstico y Estadístico de los Trastornos Mentales, DSM-IV-TR- ( 2002), este trastorno tiene una incidencia del 3-6\% en la población normal.

Desde que Still (1902) realizase la primera aproximación al trastorno, hablando de un déficit en el control moral, han sido distintas las aproximaciones que ha tenido el trastorno, como hiperkinesia, disfunción cerebral mínima (Clements, 1966), o más tarde Virginia Douglas (1972) argumentó que la deficiencia básica de los niños hiperactivos no era el excesivo grado de actividad, sino su incapacidad para mantener la atención y su impulsividad.

Así mismo, las consecuencias personales y sociales de la impulsividad son evidentes dado el alto índice de niños que cada día son diagnosticados bajo el TDAH, una manifestación extrema de las dificultades relacionadas con la autorregulación.

Aunque en las últimas décadas se ha conceptualizado el TDAH como un trastorno de las funciones ejecutivas, autores como Banaschewski, Hollis, Oosterlaan, Roeyers, Rubia, Willcutt y Taylor (2005), señalan que este trastorno podría estar más relacionado con anormalidades motivacionales, desorganización motriz y descontrol temporal. Otros autores, como Sonuga-Barke (2005) o Mclnerney y Kerns (2001), ven en la motivación un aspecto esencial para explicar y comprender el TDAH.

Sin embargo, las primeras aproximaciones al estudio de la motivación humana se basaban en términos como activación, instinto, impulso, energización; la necesidad de 
explicar por qué un individuo comenzaba, continuaba o terminaba una tarea, o sea, aquello que motiva o "empuja" a un sujeto a llevar a cabo determinadas acciones, su motivación (Douglas, 1972).

En este sentido, es importante diferenciar entre motivación extrínseca e intrínseca en el sujeto. La primera, vendrá determinada por refuerzos externos o recompensas que variarán su significación según los valores de cada persona. En cambio, la motivación intrínseca está impulsada por ganas e interés propio por involucrarse en una tarea por el simple hecho de participar, con tendencias hacia el aprendizaje y la creatividad. La motivación intrínseca es muy importante para el desarrollo cognitivo y social del niño; y además, según Romero Ayuso (2006), este correcto desarrollo motivacional es la principal fuente de exploración y curiosidad a lo largo de la vida.

Por todo ello, cada vez es mayor el interés por saber el papel que juega la motivación en los niños con TDAH. Es importante adoptar una visión global para la explicación del trastorno y deben incluirse factores afectivos, emocionales y sociales que inciden en su desarrollo. En esta línea, algunas investigaciones como las de Miranda (2002) o Keown y Woodward (2002), señalan que el contexto familiar tiene un papel fundamental en el curso del TDAH.

La disfunción familiar puede constituir un factor de riesgo que interactúa con la predisposición del niño, y exacerbar la presentación de los síntomas y su continuidad, (Miranda García, 2002). En este marco, los factores familiares no se consideran como la causa original del TDAH, pero sí se cree que mantienen e incluso amplifican la sintomatología de éste en el curso del desarrollo. En esta línea, Keown y Woodward, (2002), han tratado de analizar las variables predictoras de los problemas conductuales de los niños hiperactivos, a partir del análisis de las relaciones de los padres con sus hijos hiperactivos en edades tempranas en comparación con las relaciones padres-niños sin TDAH. Los resultados obtenidos de diversos estudios indican que los padres de los niños hiperactivos utilizan prácticas disciplinarias laxas, tienen menos comunicación con sus hijos y, además, hay una menor sincronía en las interacciones madre-hijo. Los hallazgos en el estudio de Miranda (2002), sugieren que la forma en que interactúan los padres con sus hijos hiperactivos en edades preescolares es precursora del desarrollo de dificultades de comportamiento experimentado por los niños hiperactivos en edades posteriores.

\section{Causas del TDAH}

\section{Factores genéticos}

Actualmente los niños empiezan a manifestar los síntomas de hiperactividad e inatención a partir de los 3-4 años, estos se reducen en la adolescencia y persisten en la edad adulta, (García, 2009). Como consecuencia de un deficiente desarrollo embrionario o genético, los niños con TDAH manifiestan una gran dificultad para guiar sus conductas mediante autoinstrucciones y para frenar sus propias conductas inapropiadas.

Para ello, existen estudios, como ejemplo el de Clements (1966), que demuestran que el hecho de que la madre fume en el embarazo multiplica por tres el riesgo de que el bebé desarrolle TDAH en sus primeros años de vida. Igual ocurre con el consumo de alcohol y de otras drogas durante la gestación, que también puede tener otras repercusiones cognoscitivas en el desarrollo del niño. 


\section{Factores psicobiológicos}

Los datos sugieren que la mayor pendiente del gradiente de refuerzo podría explicar la impulsividad y la hiperactividad. Sagvolden y Sergeant (1998) consideran que éstas son consecuencias de una alteración en el intervalo del gradiente de refuerzo (intervalo entre respuesta y refuerzo), que tiene una pendiente más pronunciada de lo normal.

El suceso de un estímulo gratificante puede reforzar la conducta que lo acaba de preceder. Los estímulos reforzantes son más eficaces si siguen inmediatamente a una conducta: cuanto mayor sea el aplazamiento, menos eficaz será el refuerzo. Sagvolden y Sergeant (1998), sugieren que ciertas deficiencias de la transmisión dopaminérgica en sujetos con TDAH aumenta la inclinación de la pendiente de gradiente de refuerzo, lo que significa que el refuerzo inmediato es aún más eficaz en estos niños.

La mayoría de investigaciones señalan que el TDAH se debe a anomalías de una red neural de regiones cerebrales que incluyen el estriado (núcleo caudal y putamen) y la corteza prefrontal (que tiene conexiones recíprocas con el estriado). Otra región también implicada en pruebas cognoscitivas en adultos es la corteza cingulada anterior. Según Sagvolden y Sergeant (1998), esta región modula la selección de estímulos y media la selección de respuesta, desempeñando un papel central en el proceso de atención; además encontraron hipoactivación de estas estructuras en el cerebro de personas con TDAH mientras realizaban tareas que precisaban atención minuciosa y capacidad de inhibir una respuesta.

\section{Factores ambientales}

No puede olvidarse las interacciones que operan entre los factores orgánicos y ambientales.

Según Miranda y Soriano (2010), la sociedad ha experimentado en este siglo cambios sociales radicales que han creado unas condiciones sociales que intensifican los síntomas del TDAH. Por ejemplo, el trabajo sedentario que exige una atención mental prolongada, la gratificación instantánea que proporciona la tecnología electrónica, las altas tasas de divorcios y familias no normativas, y el estrés que supone para las familias el ejercicio profesional de ambos padres, entre otros.

Por lo tanto, la calidad de las relaciones familiares y la competencia parental pueden jugar un papel importante, no sólo en la probabilidad de remisión clínica, sino también en la severidad y comorbilidad del desorden, la respuesta al tratamiento y el pronóstico.

\section{Características del TDAH manifestadas en la conducta}

Entre otras características presentes en el TDAH, se enumeran a continuación las más comunes manifiestas en la conducta diaria en niños diagnosticados de este trastorno (Kerns, Mclnerney y Wilde, 2001):

1. Muestran una incapacidad muy limitante para la inhibición de conductas.

2. Presentan una actividad motriz incesante, inadecuada e inoportuna, que resulta inadaptativa. 
3. Significativa dificultad en el control de la atención: dificultades en atención sostenida, en atención selectiva y en la atención dividida.

4. Impulsividad y déficit en procesos de análisis y síntesis, primero actúa y después piensa.

5. Memoria de trabajo: presentan menor eficacia en la codificación de los estímulos. Se les cataloga de "olvidadizos" y de que "sólo viven en el presente" sin prestar atención a las consecuencias futuras.

6. Autorregulación de la motivación y el afecto: muestran intereses numerosos y dispares, pero de muy corta duración.

7. Internalización del lenguaje: facilita la guía y el control de la conducta, los alumnos con TDAH poseen retraso en ese aspecto.

8. Dificultades en el aprendizaje e inadaptación escolar, familiar y social: aumenta la tensión en la clase, la ansiedad en los maestros y el rechazo a estos alumnos. También en el hogar y en otras situaciones su incapacidad para el control ejecutivo les acarrea inadaptación.

Todas estas características influyen significativamente en el desempeño académico y social de los niños que presentan el trastorno. Tan importante son las características impulsivas como las hiperactivas, así como las que se refieren al control y guía de la conducta (derivadas de su déficit de atención). El niño/a diagnosticado o no de esta alteración manifiesta una incapacidad sustancial para adaptarse a un normal y adecuado desarrollo, lo que aumenta la posibilidad de que se presenten anomalías asociadas (tensión en la clase, ansiedad en los maestros, etc.).

\section{Métodos y estrategias para trabajar la motivación en alumnos con TDAH}

Como alternativa o complemento a los tratamientos farmacológicos, las intervenciones psicosociales constituyen opciones útiles para el manejo de los síntomas centrales del TDAH, así como para abordar los múltiples problemas asociados que presentan los niños con este trastorno.

Frente a las demandas escolares que presentan los niños con TDAH en el aula, se presentan algunas estrategias didácticas para regular la motivación y las emociones en estos niños.

Una de estas demandas es el interés por el aprendizaje, en el que el problema del niño es la poca o nula motivación intrínseca. Para ello, se proponen algunas estrategias didácticas, tales como (Angrino, Panesso y Valencia, 2010):

1. Promover la participación del niño en el aula (ayudante de la instrucción, escribir palabras claves en la pizarra, recoger cuadernos, borrar la pizarra, realizar juegos de roles, etc.).

2. Explicitar los objetivos de aprendizaje de cada actividad.

3. Relacionar los contenidos con los intereses del alumno.

4. Proponer metas a corto plazo.

5. Modificar frecuentemente las características de la tarea.

6. Proponer actividades que garanticen el éxito, evitando la frustración y el abandono.

7. Utilizar programas de economía de fichas y/o contratos de contingencia. 
Otra de las demandas escolares es el autocontrol emocional, en la que el problema del niño con TDAH es el insuficiente control de las emociones. Las estrategias didácticas que se proponen son (Klimenko, 2011):

1. El entrenamiento en habilidades sociales.

2. Proponer tareas que se ajusten a sus intereses.

3. Proponer tareas que desarrollen su responsabilidad.

4. Brindar feedback sobre sus emociones para que reflexione sobre sí mismo y sus conductas consecuentes.

Por último, la demanda del locus de control interno, en la que el problema del niño es el sesgo atribucional. Se proponen diversas estrategias didácticas, como por ejemplo (Klimenko, 2011):

1. Dar claves para que pueda controlar su entorno.

2. Brindar información constructiva sobre sus errores.

3. Procurar interacciones satisfactorias.

En consecuencia, hay que evitar hacer comentarios sobre conductas indicativas de falta de atención como "estás en las nubes" o dar órdenes como "no te distraigas con el lápiz". Es mejor suministrar al alumno claves verbales discriminativas que le animen a reflexionar sobre su forma de comportarse, como "¿estás terminando ya tu trabajo?". Cuando se equivoque será conveniente dar al niño una oportunidad para que pueda intentarlo otra vez, procurar que tenga éxito proporcionándole las ayudas necesarias y alabarle por ello (Siegenthaler, Herrero y Muñoz, 2011).

\section{Conclusiones}

La dificultad de encontrar una relación firme entre el TDAH y la motivación radica en que la mayoría de los estudios, como los de Sonuga-Barke (2005) o Mclnerney y Kerns (2001), sostienen la intervención de otros factores como atribuciones, locus de control, feedback, refuerzos, etc., además también ha influido la escasa relevancia que se le ha otorgado a la motivación en el estudio del TDAH, junto con la carencia de estudios desde sus comienzos en las distintas investigaciones.

Todo ello, manifiesta la necesidad de incluir la dimensión motivacional en los estudios sobre el trastorno con el fin de mejorar las estrategias utilizadas en los sujetos con TDAH, posibilitando así el aumento o mejora en el rendimiento escolar.

En definitiva, este trabajo justifica la importancia de nuevas investigaciones que incluyan la motivación como factor primordial en el rendimiento académico de niños con TDAH, la necesidad de intervenciones psicosociales para la población afectada y su entorno inmediato, priorizar estrategias de autocontrol en los alumnos como alternativa y/o complemento valioso a los psicofármacos. Además, cualquier tipo de intervención con niños de TDAH debe incluir no sólo una valoración de los componentes cognitivos, sino también de los psicosociales como el entorno social, el tipo de feedback verbal que reciben, tipo de tareas, fomento de competencias necesarias, establecimiento de reglas, autocontrol e integración social. 


\section{Bibliografía}

Angrino, S. O., Panesso, I. C., y Valencia, Á. I. (2010). Las situaciones de resolución de problemas como estrategia de trabajo con niños diagnosticados con TDAH. Pensamiento Psicológico, 2(7).

Ayuso, D. M. R. (2006). La motivación y el rendimiento ocupacional en niños con trastorno por déficit de atención con hiperactividad. Revista electrónica de terapia ocupacional Galicia, TOG, $(4), 8$.

Banaschewski, T., Hollis, C., Oosterlaan, J., Roeyers, H., Rubia, K., Willcutt, E., y Taylor, E. (2005). Towards an understanding of unique and shared pathways in the psychopathophysiology of ADHD. Developmental Science,8(2), 132-140.

Clements, S. D. (1966). Minimal Brain Dysfunction in Children: Terminology and Identification: Phase One of a Three Phase Project. Washington DC: US Department of Health, Education and Welfare.

Douglas, V. I. (1972). Stop, look and listen: The problem of sustained attention and impulse control in hyperactive and normal children. Canadian Journal of Behavioural Science, 4(4), 259.

García-Castellar R., Miranda-Casas A., Siegenthaler-Hierro R. y Jara-Jiménez, P. (2006). Impacto familiar de los niños con trastorno por déficit de atención con Hiperactividad subtipo combinado: efecto de los problemas de conducta asociados. PREV NEUROL 42 (3), 137-143.

Keown L.J. y Woodward L.J. (2002). Early parent-child relations and family functioning of preschool boy with pervasive hyperactivity. J Abnorm Child Psychol, 30, 541-53.

Kerns, K. A., Mclnerney, R. J., y Wilde, N. J. (2001). Time reproduction, working memory, and behavioral inhibition in children with ADHD. Child Neuropsychology, 7(1), 21-31.

Klimenko, O. (2011). La enseñanza de las estrategias cognitivas y metacognitivas como una vía de apoyo para el aprendizaje autónomo en los niños con déficit de atención sostenida. Revista Virtual Universidad Católica del Norte, 1(27).

Manassero, M. y Vazquez A., (1998). Validación de una escala de motivación de logro. Psicothema, 10(2), 333-351.

Miranda A., García R., Presentación MJ. (2002): Factores moduladores de la eficacia de una intervención psicosocial en niños con trastorno por déficit de atención con hiperactividad. Rev Neurol 34 (Supl 1): S91-7.

Miranda Casas, A. y Soriano Ferrer, M. (2010). Tratamientos Psicosociales Eficaces para el Trastorno por Déficit de Atención con Hiperactividad. Información psicológica, (100), 100-114.

Oosterland, J., Logan, G.D y Sergeant, J.A (1998). Response inhibition in AD/HD, CD, comorbid $A D / H D+C D$, anxious, and control children: A meta-analysis of studies with the stop task. Journal of Child Psychology and Psychiatry, 3, 411-425.

Siegenthaler, R., Herrero, P. y Muñoz, J. (2011). Estrategias didácticas inclusivas en TDAH.

Sonuga-Barke, E. J. (2005). Causal models of attention-deficit/hyperactivity disorder: from common simple deficits to multiple developmental pathways. Biological psychiatry, 57(11), 1231-1238.

Still, G. F. (1902). Some abnormal psychical conditions in children. Lancet,1(1008), 1077-1082. 